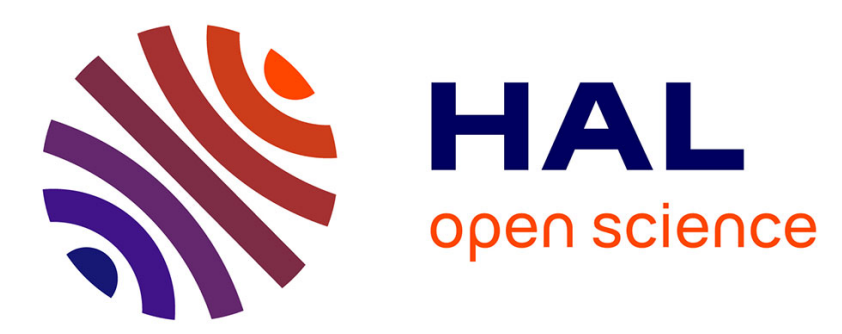

\title{
Development and first use of an experimental device for fission-induced spectrometry applied to neutron flux monitoring
}

Maxime Lamotte, G. de Izarra, C. Jammes

\section{- To cite this version:}

Maxime Lamotte, G. de Izarra, C. Jammes. Development and first use of an experimental device for fission-induced spectrometry applied to neutron flux monitoring. Nuclear Instruments and Methods in Physics Research Section A: Accelerators, Spectrometers, Detectors and Associated Equipment, In press, 953, pp.163236. 10.1016/j.nima.2019.163236 . cea-02932104

\section{HAL Id: cea-02932104 https://hal-cea.archives-ouvertes.fr/cea-02932104}

Submitted on 7 Sep 2020

HAL is a multi-disciplinary open access archive for the deposit and dissemination of scientific research documents, whether they are published or not. The documents may come from teaching and research institutions in France or abroad, or from public or private research centers.
L'archive ouverte pluridisciplinaire HAL, est destinée au dépôt et à la diffusion de documents scientifiques de niveau recherche, publiés ou non, émanant des établissements d'enseignement et de recherche français ou étrangers, des laboratoires publics ou privés. 


\title{
Development and first use of an experimental device for fission-induced spectrometry applied to neutron flux monitoring
}

\author{
M. LAMOTTE ${ }^{\mathrm{a}}$, G. DE IZARRA ${ }^{\mathrm{a}}$, C. JAMMES ${ }^{\mathrm{a}}$ \\ ${ }^{a}$ CEA, DEN DER, Instrumentation Sensors and Dosimetry Laboratory, Cadarache, \\ F-13108 Saint-Paul-lez-Durance, France.
}

\begin{abstract}
In order to provide dependable neutron flux instrumentation for Generation IV Sodium-cooled Fast Reactors (SFR), the French Atomic and Alternative Energies Commission (CEA) is investigating the feasibility of an innovative technology based on the optical signal produced within a fission chamber. In such gaseous detectors, neutrons interact with fissile material releasing heavy ions in the MeV-range, eventually leading to spontaneous photon emission in the ultraviolet-to-infrared range thanks to gas excitation and ionization. In this paper, the process of light generation is analyzed through semi-empirical models and custom-developed codes. A specific instrumentation has been defined and manufactured to emulate optical fission chambers and collect basic spectroscopic data required for model-based development approach. That testing device was named PSEG, which stands for Prototype of Scintillator Electrodes-Gas. Experimental validations have been carried out: the ionized argon gas exhibited no local thermodynamic equilibrium. Furthermore, the broadening of selected argon emission lines due to pressure change offers a promising self-diagnosis capability for future optical fission chambers.

Keywords: fission chambers, radiation-hard detectors, gaseous detectors, gas scintillation
\end{abstract}

PACS: 29.85.-cAMODIF, 28.50.Dr, 28.41.Rc

Preprint submitted to Nuclear Instruments and Methods Section A 


\section{Introduction}

Nuclear energy technology has not only to meet safety requirements but also relies on dependable instrumentation for reactor control and protection [1]. Because the neutron flux monitoring instrumentation of a pool-type Sodiumcooled Fast Reactor (SFR) of Generation IV has to be placed into its vessel, robust fission chambers are required. Only high-temperature fission chambers have proven to sustain and reject the signal induced by up to $10 \mathrm{MGy} / \mathrm{h}$ of gamma irradiation as found behind a neutron shield[2]. Placement of such detectors in an SFR pool therefore requires a high neutron-to-gamma discrimination level. At high temperature above $400^{\circ} \mathrm{C}$, standard fission chambers endure partial discharges, occurring in both the sensor and insulated cables [2], and challenges neutron detection at startup [3]. Ability for in-vessel neutron detector to self-diagnosis is also a key feature for dependable measurements. The French Alternative Energies and Atomic Energy Commission (CEA) proposes a new generation of neutron detectors for SFR neutron flux monitoring, based on the luminescence of noble gases. This optical fission chamber requires no power on the sensor, while the signal can be transmitted with optical fibers over a long distance in the harsh environment of such a reactor type. It is important to note, that the development of such a detector technology is enabled by recent advances in optical fiber technology applied to nuclear reactor instrumentation [4] [5] [6] and latest progresses on high sensitive near-infrared optical instrumentation [7].

No need for polarization of optical fission chambers suppresses the partial discharge risk and also enables pressure diagnosis by means of light-emission spectra analysis, while preserving high gamma-to-neutron discrimination. Transmitting information by optical means cancels requirements for electric insulation and electromagnetic immunity. In addition, optical fibers make it possible to multiplex signals in a single conductor, leading to an easier wiring through the upper plate of a nuclear reactor vessel.

This paper starts with the state of the art of both optical neutron detector 
tentatives and available spectroscopic data. Methodology and models used to assess the feasibility of a gas-scintillation-based neutron detector for nuclear reactor will be then detailed. The definition and manufacture of our testing device dedicated to noble gas excitation study will be also presented. Report of experiments validating our developed testing device will follow. Conclusion obtained from our observations and future milestones towards development of optical fission chambers and cold-plasma models will be finally exposed.

\section{State of the art}

Since the radium discovery, the luminescence of rare gas under heavy ion irradiation [8] [9] have been observed. Koch at CEA utilized noble gas scintillation for neutron flux instrumentation in the year 1959 [10]. Through her extensive work on the EL-2 reactor, an optical setup for neutron detection featured promising performances such as nanosecond-range time resolution, satisfactory particle energy discrimination, efficient gamma-ray rejection and thermal-tofast neutron energy detection range. The active part, which was dipped into the reactor pool, was an argon-filled silica vial enclosing a uranium foil. A fluorescent layer coating that vial made visible far-ultraviolet photons coming from both recombinations and decays of argon excited-states. A several-meter-long light-guide tube going out of the pool was ended by mirrors leading photons to a Photo-Multiplier Tube (PMT). That work was abandoned due to the degradation of the wavelength-shifter under gamma irradiation, and the difficulty to transport UV-light from the reactor core to a shielded remote location. Since Koch's work, advent of radiation-hardened optical fibers and solid-state photon detectors happened, allowing nowadays remote near-infrared photon sensing with high efficiency and over long distance. High pressure heavy-ion-induced plasmas are not as well described and modeled as other plasmas (e.g. corona or magnetron). Because no Local Thermodynamic Equilibrium (LTE) should be encountered in such weakly ionized plasmas, general models have to be used in order to understand mechanisms and compute the expected emission spec- 
trum generated in an optical fission chamber. Miley, Thiess and Guyot [11, 12] computed energy deposition of various heavy ions in rare gases using $\mathrm{W}$-value, the mean energy needed to create an ion-electron pair and simple models, but neither theoretical spectrum nor luminous intensity estimates were obtained. Optical Emission Spectroscopy analysis of heavy-ions induced scintillation can provide Electron Energy Distribution Function (EEDF). This way, one will be able to simulate plasma kinetics.

\section{Modeling of fission-induced photon detection}

Because the neutron irradiation of a bulky experimental device requires nuclear-grade materials, radio-protection provisions and size limitations, liminary experiments will be more conveniently conducted with an alpha-emitter as source of heavy ions instead of a fissile coating in a dedicated plasma reactor. Although alpha particles are lighter and then release less energy $(\sim 5 \mathrm{MeV})$ than fission fragments $(\sim 100 \mathrm{MeV})$, they however display the same energy amount for ion-pair formation (W-value) in rare gases [13] and have a similar range (36 $\mathrm{mm}$ against $24 \mathrm{~mm}$ ). In addition, the maximum energy transfer during electron/ion collisions matches the order of magnitude of that found for fission fragments $(\sim 3 \mathrm{keV})$. This way, it is expected that the primary electron energy distribution is close to that obtained with fission fragments. Hence, although a lower energy deposition rate will lead to a weaker overall photon amplitude, alpha particles are representative enough of fission fragments for gas excitation and ionization. Hereafter is described the methodology of defining our testing device, the Prototype of Scintillator Electrodes-Gas (PSEG) dedicated to Optical Emission Spectroscopy (OES). PSEG definition follows the flowchart displayed in Fig. 1. Future simulations of optical fission chambers will rely on models established by observations obtained on PSEG. Because operating scenarios will involve various noble gases and gas excitation processes, PSEG has to be versatile in terms of pressure, filling gas, excitation source and instrumentation. This way, PSEG (Fig. 4) will bring information on the distribution and 
evolution of gas excited states and also benchmark optical detection sensor solutions such as Avalanche Photo-Diode (APD), Silicon Photo-Multiplier (SiPM) and Charge Coupled Devices (CCD).

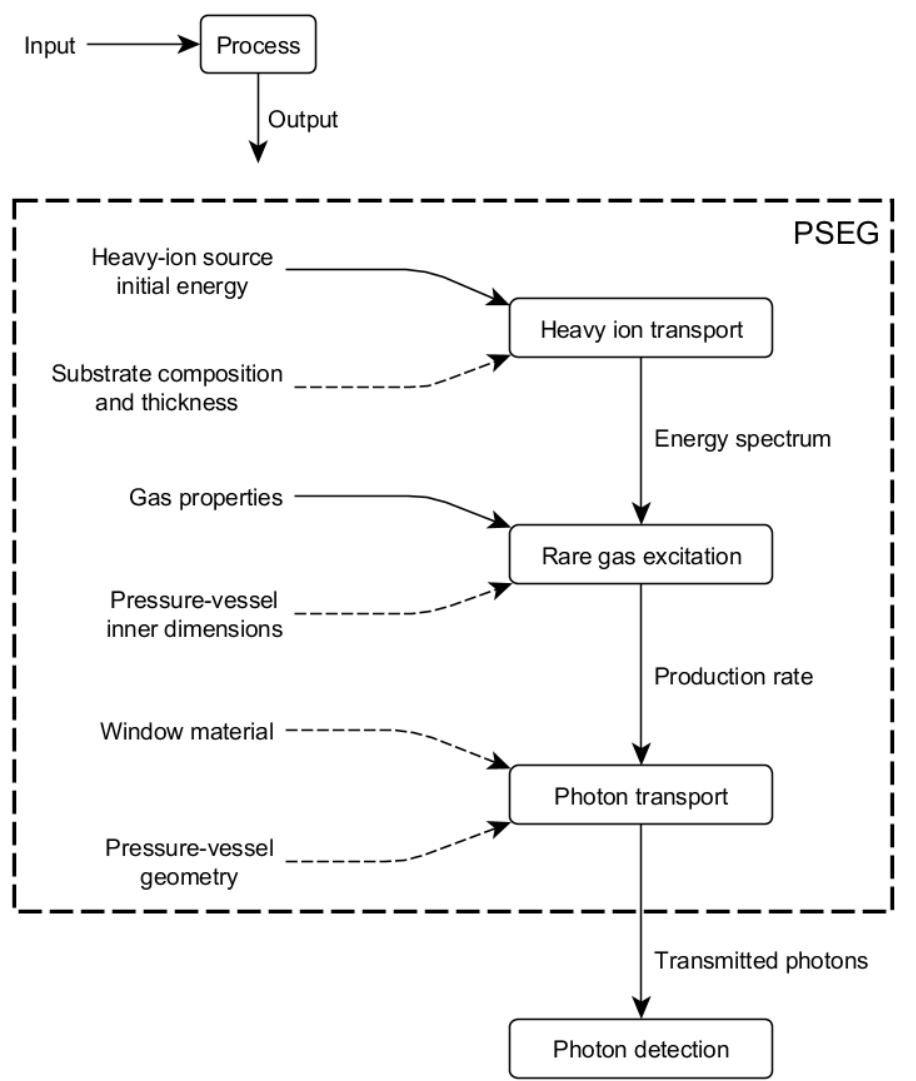

Figure 1: Flowchart of PSEG definition steps. Dashed arrows indicates variables to be estimated for the device using analytical models.

\subsection{Heavy-ion transport in coating}

To ensure a high level of gas excitation by heavy ions featuring a narrow energy distribution, an alpha-emitter plutonium 238, with a half-life of 90 years was selected. In a fissile layer, $\mathrm{MeV}$-range heavy ions produced at random depths endure slowing down through collisions, described by the so-called moderation law of Eq. 1. 


$$
E(x)=E_{0}\left(1-\frac{x}{R_{E_{0}}}\right)^{n}
$$

This semi-empirical model, as used by Chung and Prelas [14], describes the energy $E$ of a particle born at a given distance $x . R_{E 0}$ is the range of particles with initial energy $E_{0}$ in a layer of a given density $\rho$. The slowing down parameter $n$ is estimated by fitting the moderation law to either experimental data [17] [16] or tabulated data provided by the well-known SRIM code [15]. Table 1 displays the fitting parameter $n$ for alpha excitation sources and fission fragments produced in fissile coatings.

Given that the coating thickness slows down ions, its value comes out of a tradeoff between a high ion-source strength and high ion energies. For a $\sim 55 \mathrm{MeV}$ alpha particle, the range within a plutonium oxide coating is about $11 \mu \mathrm{m}$. We computed the energy distribution of heavy ions emerging from the plutonium oxide coating using a Monte Carlo approach and Eq. 1 for initial random depths and emission directions. The ion track is assumed as straight. The ion slowing down is estimated using a pre-determined $n$ value. Figure 2 shows the average energy of alpha particles escaping a plutonium coating.

Table 1: Slowing-down in various coatings and 1-atm argon. HFF stands for Heavy Fission Fragment and LFF for Light Fission Fragment.

\begin{tabular}{|c|c|c|c|c|c|c|}
\hline \multicolumn{6}{|c|}{ Coating } & \multirow{2}{*}{$\begin{array}{l}\text { Argon } \\
\text { Range } \\
(\mathrm{mm})\end{array}$} \\
\hline Material & Ion & $\begin{array}{c}\text { Mass } \\
(\mathrm{AMU})\end{array}$ & $\begin{array}{l}\text { Initial energy } \\
\qquad(\mathrm{MeV})\end{array}$ & $\begin{array}{l}\text { Range } \\
(\mathrm{mm})\end{array}$ & $n$ & \\
\hline${ }^{238} \mathrm{Pu}$ & $\alpha$ & 4 & 5.56 & 12.0 & 0.74 & 36.3 \\
\hline${ }^{10} \mathrm{~B}$ & $\alpha$ & 4 & 1.49 & 3.41 & 1.25 & 11.1 \\
\hline${ }^{235} \mathrm{U}$ & $\mathrm{HFF}$ & 139 & 68.1 & 9.67 & 1.65 & 19.4 \\
\hline${ }^{235} \mathrm{U}$ & LFF & 95 & 98.9 & 7.42 & 1.69 & 23.9 \\
\hline
\end{tabular}



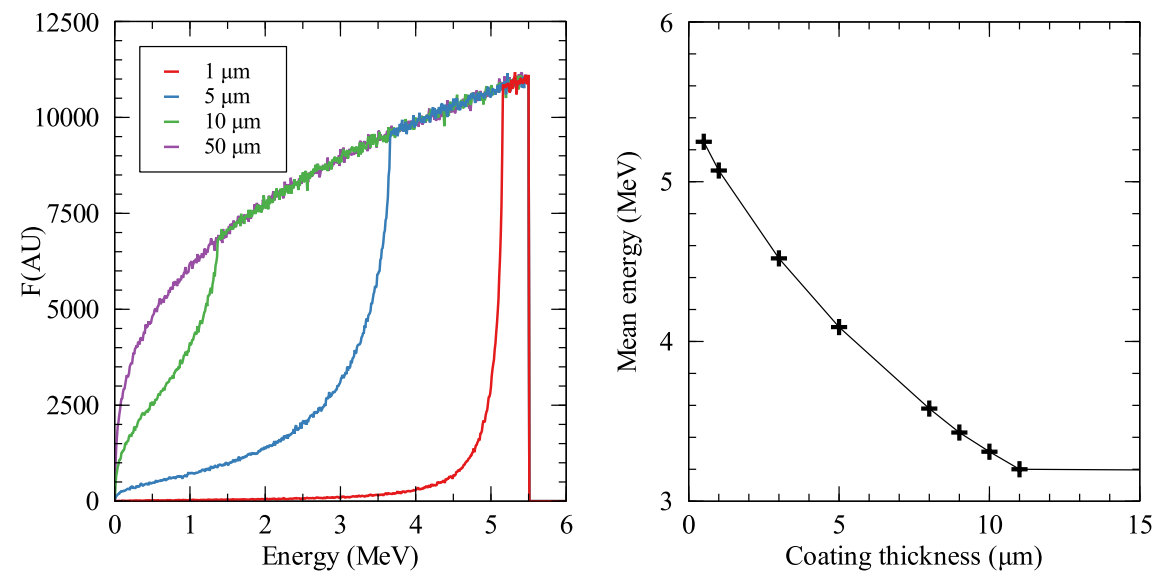

Figure 2: Ion energy distribution and a ${ }^{238} \mathrm{Pu}$ coating thickness. Energy distribution of alpha particles escaping a coating of various thicknesses (left). Mean energy of alpha particles as a function of coating thickness (right).

\subsection{Rare gas excitation}

Gas ionization by heavy particles is often grossly modeled with the use of the so-called $W$-value, the mean energy needed to produce an ion-electron pair. All phenomena arising from collisions and electronic cascades are taken into account in this quantity [18]: secondary electron emission, Penning ionization, atomic level excitation. However, hereafter is more relevant to use the mean energy needed to excite an atom, denoted as $W_{\text {exc }}[19]$ :

$$
W_{\mathrm{exc}}=\left(\frac{N_{\mathrm{ion}}}{N_{\mathrm{exc}}}\right) E_{\mathrm{ion}}+E_{\mathrm{exc}}+\left(\frac{N_{\mathrm{ion}}}{N_{\mathrm{exc}}}\right) \epsilon
$$

$N_{\text {ion }}$ and $N_{\text {exc }}$ are the number of ions and that of excited states produced by the ions themselves. $E_{\text {ion }}$ and $E_{\text {exc }}$ are the energies of ionization and excitation by electrons. $\epsilon$ is the sub-excitation kinetic energy of delta rays. It was shown that $W$-value $\approx 0.4 \times W_{\text {exc }}[20]$. Tabulated and experimental values from [18] were used, yielding a $W_{\text {exc }}$ value of about $67.9 \mathrm{eV}$.

Assuming that a single alpha particle can excite over $E_{0} / W_{\text {exc }}$ gas atoms along its path allows us to estimate the ultimate light emission intensity. Since $W_{\text {exc }}$ has to do with the whole number of excited gas atoms only, the identification 
of atom excitation levels will have to be performed by experiments on PSEG.

Table 1 exemplifies the ranges of heavy ions for a future optical fission chamber. Lowering the detector pressure necessarily leads to increase the ranges of heavy ions that may then collide the chamber walls. We estimated the ion energy release using a Monte-Carlo integral calculation. Any mono-energy heavy ion is emitted at a given distance from the center of the disk-shaped coating, along a straight path of random hemispherical directions. Its range is described by the Bragg curve computed by SRIM. In the case when a particle hits a chamber wall, its remaining energy $E_{\text {hit }}$ is subtracted from its initial one. Then, a random propagation direction of generated photons is selected until they hit a wall or viewport. Photons hitting a viewport are finally registered to estimate the amount of light that a photo-sensor can detect. Fig.3 displays the generatedto-recovered photon ratio as a function of the PSEG pressure and diameter.
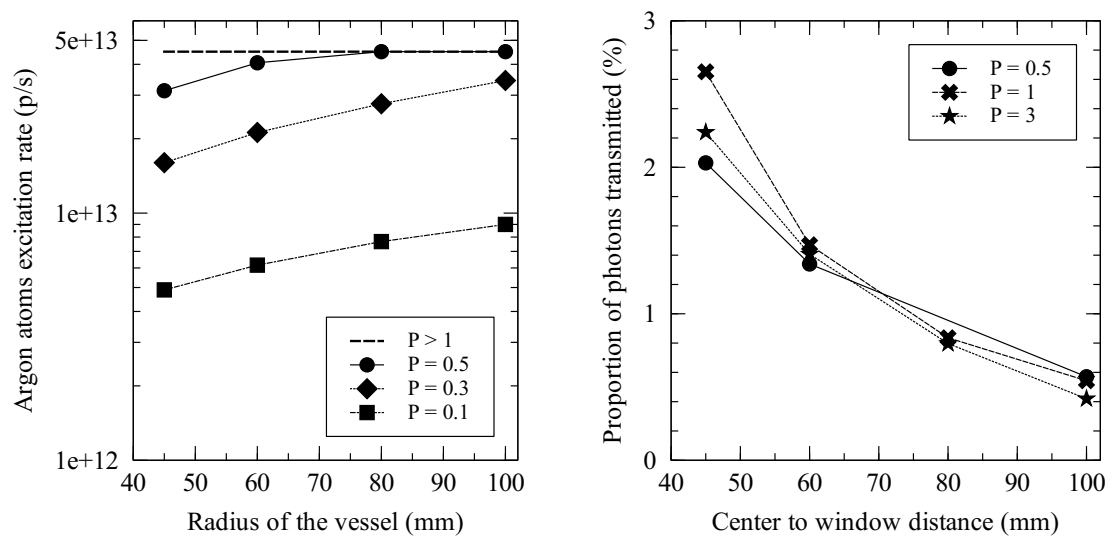

Figure 3: Argon excitation and light detection. At left, it is shown the production rate of excited argon atoms by a $1 \mathrm{E} 9-\mathrm{Bq}$ alpha source as a function of the chamber radius for various pressures (atm). At right, it is shown the proportion of photons crossing a 30-mm-diameter window to those generated within the chamber as a function of the distance between the window and alpha-emitting plate for various pressures as well. 


\section{Instrumentation definition and fabrication}

\subsection{Ionization source}

To ensure a low dispersion of alpha particle energy spectrum, a thin yet active layer of alpha-emitter has to be defined. ${ }^{238} \mathrm{Pu}$ has a high specific activity $\left(\sim 5.58 \mathrm{E} 11 \mathrm{~Bq} / \mathrm{g}\right.$ in its oxide form, $\left.\rho=11.5 \mathrm{~g} / \mathrm{cm}^{3}\right)$. In the case of a thin slab, the energy deposition rate in gas is given by:

$$
E_{\mathrm{dep}}=E_{0} \cdot A_{\mathrm{Pu}} \cdot \frac{1}{2}
$$

$A_{\mathrm{Pu}}$ is the alpha activity of the thin coating. The factor $1 / 2$ accounts for particles emitted towards the support plate.

Our $\mathrm{PuO}_{2}$ coating features $15-\mathrm{mm}$ diameter and 1- $\mu \mathrm{m}$ thickness, which are the maximum values achievable with our electro-deposition process. At least 1.1E9 $\mathrm{Bq}$ alpha particles per second should be emitted. That production rate will result in an energy release in the gas of $3 \mathrm{E} 9 \mathrm{MeV} / \mathrm{s}$. Instead, using a ${ }^{235} \mathrm{U}$ coating, a rate of $3.52 \mathrm{E} 7$ fissions per second would be required to mimic an alpha source energy release (only half of the energy is released within the gas). Such a fission rate would require a pellet of pure ${ }^{235} \mathrm{U}$ of 15 - $\mathrm{mm}$ diameter and 0.5 $\mu \mathrm{m}$ thickness irradiated by at a thermal neutron flux of $1.12 \mathrm{E} 11 \mathrm{~cm}^{2} \cdot \mathrm{s}^{-1}$ at least.

\subsection{Filling gas}

Argon was selected for its high-availability and satisfactory purity at low cost. Argon as buffer gas has to be nitrogen-free up to $100 \mathrm{ppm}$ to prevent quenching of argon metastable species by molecular nitrogen [21]. Welding application employs Arcal Prime cylinders, containing less than $10 \mathrm{ppm}$ of $\mathrm{N}_{2}$, offering a cost-effective solution for large gas consumption. Since argon is also widely-used in cold plasma physics, and its cross sections of several excitation levels are well experimentally estimated under $1 \mathrm{keV}$ [22]. The argon first excitation threshold is as low as $11.548 \mathrm{eV}$, much lower than that of helium 
( $\sim 19.82 \mathrm{eV})$, what ensures higher excitation-level populating. Since we designed a spacious ionization chamber, the complete alpha particle track is contained within the gas. This way, we can estimate energy deposition rate and plutonium layer activity. The excitation rate then reaches 4.33E13 argon atoms per second (Sect. 4.1).

\subsection{Detection window}

Both the pressure chamber optimal dimensions and geometry definition have to be consistent with placement of technical components and handling limits. Taking into account for the alpha particle range in argon at pressure between 1 and 5 bar, we chose standard $30 \mathrm{~mm}$ diameter portholes. It allows us to observe the whole plasma and makes possible reconstruction techniques like Abel inversion for spectroscopic measurements. By means of the code presented in Section 3.2, we estimated the proportion of photons crossing the viewport to generated photons. We found an optimal trade-off between the collected light, chamber radius and operational pressure (see Fig. 3).

\subsection{Mechanical structure and manufacture}

For handling and interface requirements, the chamber diameter could not be larger than $150 \mathrm{~mm}$ and welding the viewport requires a minimum distance from the chamber walls. Thus, the final design, presented in Fig. 4, comprises a $120-\mathrm{mm}$ diameter 3 -mm thick steel cylinder with two diametrically opposed circular windows welded on a 30-mm cylindrical extension. Those windows are made of fused silica that features both satisfactory transmission $(>93 \%)$ in the near-infrared region and high elastic modulus (73 GPa). An access to the chamber internal parts is achieved by means of an o'ring sealed lid made of 8-mm-thick 304L steel. Four studs are welded on the lid to support an assembly of spacers, electrodes, fissile coating and butterfly screws to ease operation in glove box. The lid also embeds two 6-mm welded pipes for gas flow as well as two BNC 3-kV feedthroughs for possibly polarizing a pair of steel electrodes. The nylon spacers, the length of which spans from 1 to $20 \mathrm{~mm}$, provide reliable 
electrode spacing while ensuring electric insulation. Such a flexible configuration is required to validate models in the case where either heavy ions collide the chamber walls or an electric field is needed.

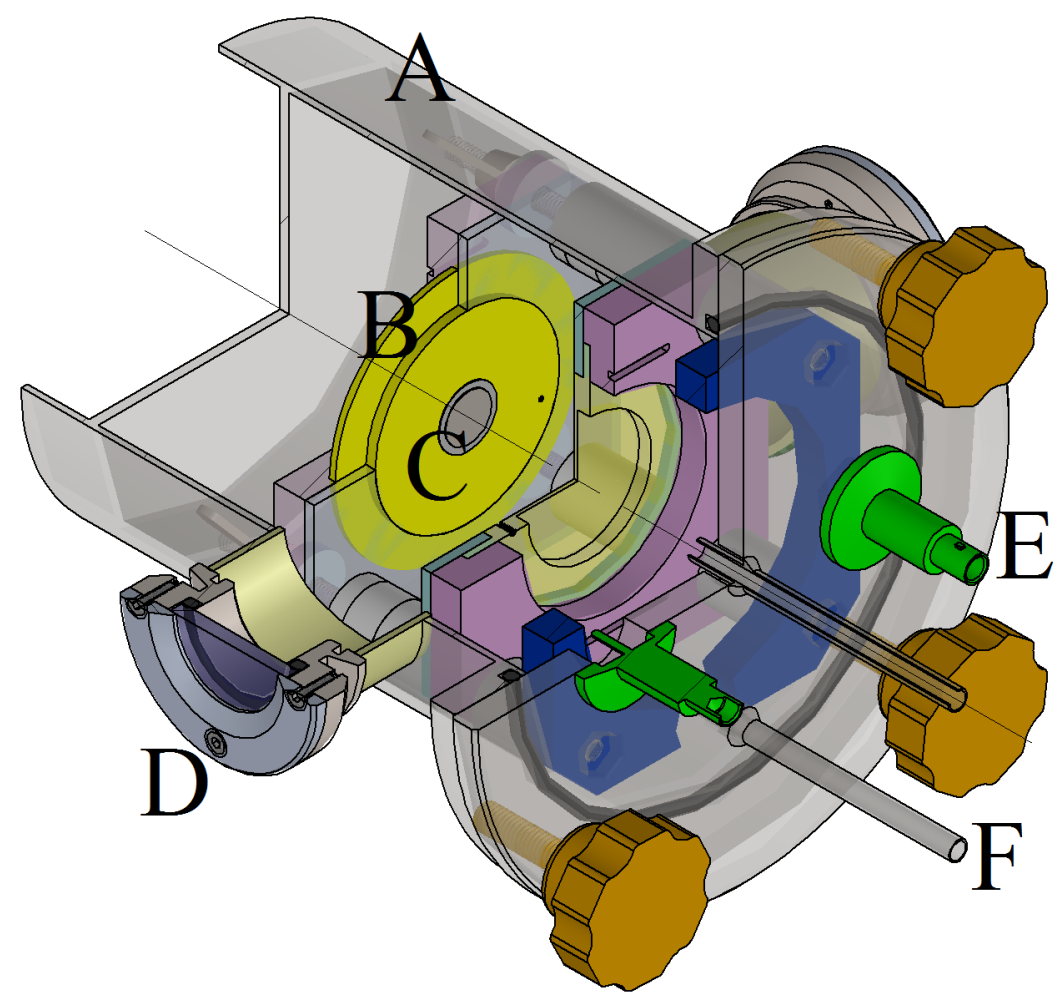

Figure 4: Cut-view of the final design of the PSEG. A: Steel envelope, B: Electrode, C: Plutonium layer D: Viewport E: BNC connector F: Gas inlet.

\subsection{Infra-red detection}

As already aforementioned, the portview was designed so that it transmits enough light for further detection. As no information is available on the emitted spectrum, one assumes that all photons have a wavelength of $800 \mathrm{~nm}$ to compute the emitted power. One then estimates a power of $50 \mathrm{nW}$ radiated light. The sensors selected for direct measurements are cooled avalanche photodiode in Geiger mode and PIN photodiode (photosensitivity of $0.5 \mathrm{~A} / \mathrm{W}$ ) 
featuring enhanced detection capabilities in the near-infrared and fast recovery time. Both of them allow for time-based analysis for future experiments. For optical emission spectroscopy, a Princeton SP2558 50-cm focal spectrograph is used with three different gratings (300,600 and 1200 grooves/mm). Spectra are recorded with a Pixis 100 BRX CCD camera featuring a $1340 \times 100$ matrix of $20 \mu \mathrm{m}$ pixels.

\section{Experimental validation}

\subsection{Sealing test}

An initial helium slow-leakage test was performed after passivation of the stainless steel parts of the chamber (Fig. 5) for vacuum retainment measurements. A gas leak rate as low as $2.17 \mathrm{E}-8 \mathrm{~Pa} \cdot \mathrm{m}^{3} \cdot \mathrm{s}^{-1}$ was detected at ultra high vacuum levels. Before initial loading with radioactive material, a positive pressure test was engaged for 2 months with $120 \%$ of nominal pressure with argon. No pressure drop was detected (gauge resolution of $100 \mathrm{~Pa}$ ).
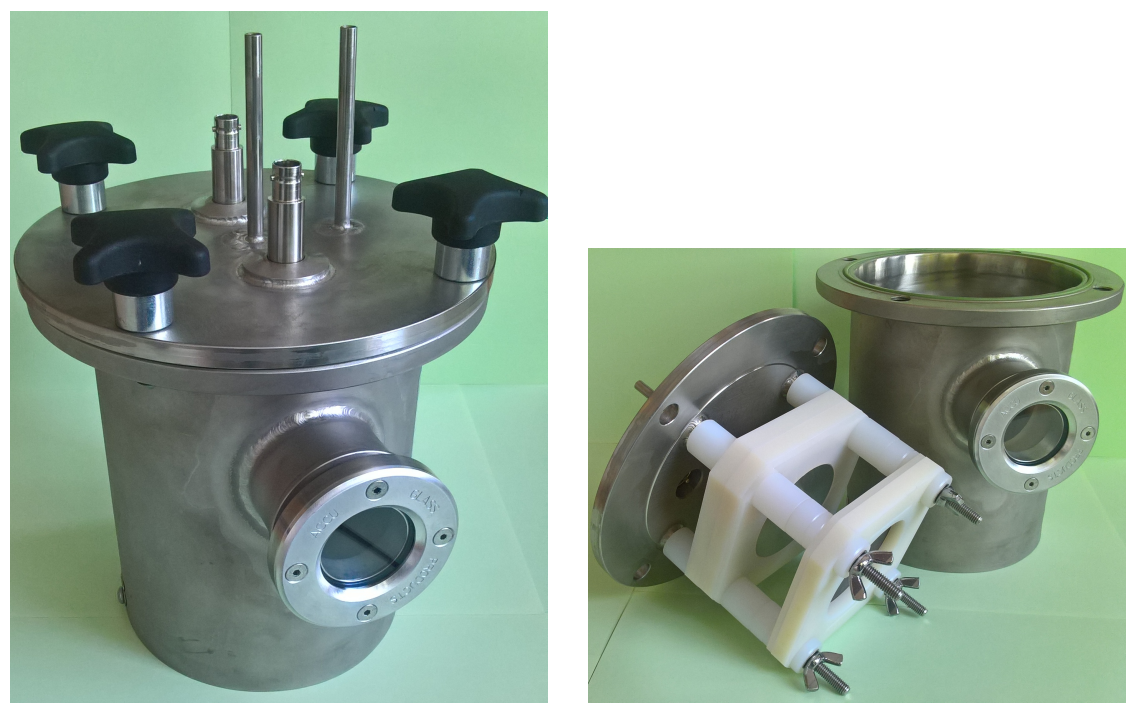

Figure 5: PSEG after machining and assembling. 


\subsection{Transmission test}

Near-infrared imaging of heavy-ion generated cold plasma helped us to precisely focus the optical assembly for spectroscopic characterization. A standard Canon 8400 digital camera, the infrared filter of which was removed, allowed us to take a photography detailed enough for qualitative definition of regions of interest (Fig. 6). A 3-minute exposure time and background-suppression processing were required.

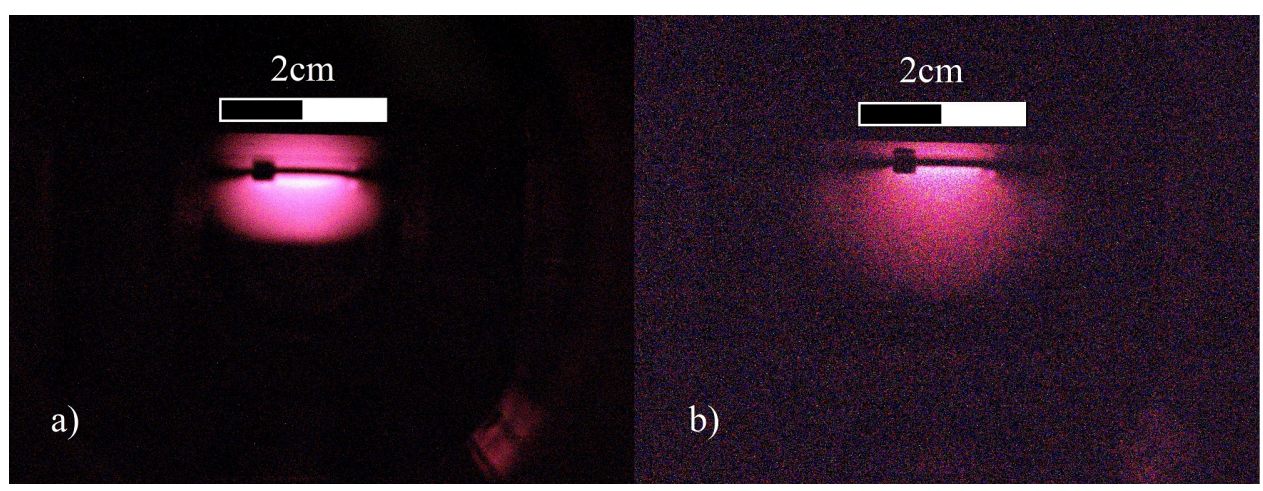

Figure 6: Images of 4 atm (a) and 2 atm (b) argon glow excited by alpha particles, as seen by a standard commercial camera without IR-filter after a 3-minute exposure time.

\subsection{Spectroscopic analysis of nuclear plasma}

A Light emission spectra analysis was performed on a 600 -grooves/mm grating blazed at $1 \mu \mathrm{m}$ and a $10 \mu \mathrm{m}$ entrance slit during a few 3-minute acquisition steps. An unfocused plasma slab $2 \mathrm{~mm}$ long located at $10 \mathrm{~mm}$ from the plutonium-layer was targeted. A near-infrared de-excitation spectrum is clearly observable, as shown in Figure 7. Neither molecular band nor continuous background is noticeable. As a result, there are no gas pollution and negligible recombinations, attachments and bremsstrahlung. A series of long acquisitions with 20-minute steps on a 1200-grooves/mm grating was performed to detect any continuum in argon radiation spectrum. No baseline after dark frame subtractions was observed. As previously noticed, one also draws the conclusion that the light emission due to atomic excitations is preponderant. Phenomena 


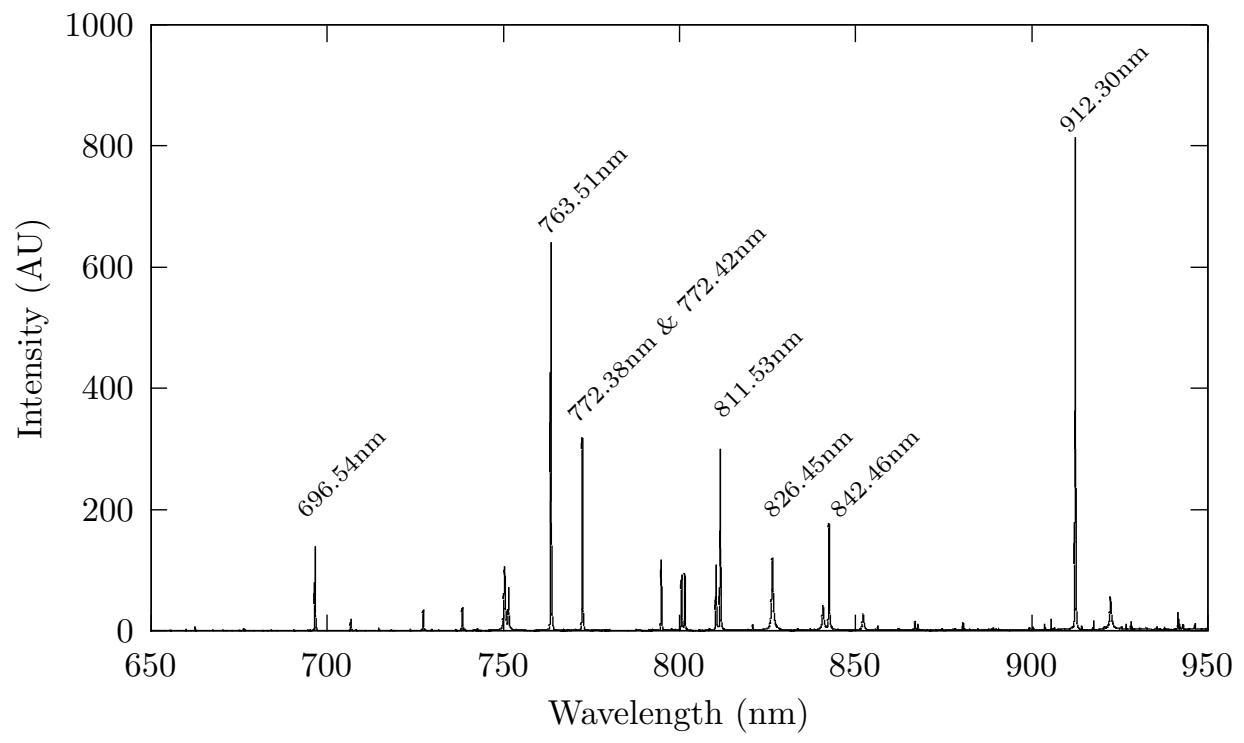

Figure 7: Spectrum obtained from PSEG filled with $2 \mathrm{~atm}$ of pure argon. No emission lines of argon ions are detected. Strong emission lines of neutral argon (Ar-I) are labeled.

such as recombinations and bremsstrahlung are negligible in excitation mechanisms as well. This way, there exist very low electron density and ionization rate. As shown in Table 2, some measured emission lines seem as affected by a pressure variation. Actually, because the argon plasma is very weakly ionized, it Table 2: Peak-area ratios of emission line as a function of PSEG argon pressure (in atm). Emission line wavelengths are expressed in $\mathrm{nm}$. The area of the 912-nm line is the reference one since it is the greatest.

\begin{tabular}{lccccc}
\hline Pressure & $912 / 922$ & $912 / 826$ & $912 / 750$ & $912 / 811$ & $912 / 763$ \\
\hline 0.1 & 6.71 & 2.82 & 0.71 & 0.72 & 0.88 \\
0.5 & 6.10 & 1.82 & 1.62 & 1.44 & 0.91 \\
1 & 5.97 & 1.75 & 1.97 & 1.59 & 0.97 \\
2 & 7.07 & 0.76 & 2.8 & 1.85 & 0.93 \\
4 & 7.42 & 0.50 & 2.59 & 1.63 & 0.84 \\
\hline
\end{tabular}


is likely that a so-called resonance broadening effect happened [23]: radiativelylinked levels decay into both the resonant levels ${ }^{1} \mathrm{~S}_{2}$ and ${ }^{1} \mathrm{~S}_{4}$. Such the observed broadening is then accounted for by argon-argon collisions only. After Ref. [23], the Full Width at Half Maximum (FWHM) of such resonance-broadened lines FWHM can be estimated using

$$
\Delta_{1 / 2}^{r}=8.6 \mathrm{E}-30 \cdot\left(g_{i} / g_{k}\right)^{0.5} \cdot \lambda^{2} \cdot \lambda_{r} \cdot f_{r} \cdot N_{i}
$$

$g_{i}$ et $g_{k}$ are respectively statistical weights of upper and lower levels of the radiative decay occurring at wavelength $\lambda$ in $\AA$. Resonance wavelength is denoted by $\lambda_{r}$ with $f_{r}$ as oscillator strength, atomic density $N_{i}$ in at.cm ${ }^{-3}$. In other words, the linearly increases w.r.t. pressure. As shown in Fig. 8, resonance broadening effect could thus be applied for pressure monitoring or out-gassing detection of an optical fission chamber. The FWHM of argon $826 \mathrm{~nm}, 922 \mathrm{~nm}$ (resonant ${ }^{1} \mathrm{~S}_{2}$ linked levels) and $912-\mathrm{nm}$ (non resonant) emission lines as a function of the PSEG pressure.

In order to better characterize the PSEG plasma, we are also interested in validating the LTE assumption. If so, the argon level population has to follow a Boltzmann law. It is then possible to write a line emission coefficient integrated over the whole line profile as

$$
\epsilon=g_{t} \frac{N}{Z(T)} \frac{h c}{4 \pi \lambda} A_{t b} \exp \left(\frac{-E_{t}}{k T}\right)
$$

where $Z(T)$ is the partition function, $N$ the number of argon atoms, $A_{t b}$ the Einstein coefficient between level $t$ and $b, g_{t}$ the degeneracy of level $t, E_{t}$ the energy of level $t$, and $T$ the temperature. That equation is then turned into a linear relationship:

$$
\log \frac{\epsilon \lambda 4 \pi}{A_{t b} h c g_{t}}=-\frac{-E_{t}}{k T}+\ln \left(\frac{N}{Z T}\right)
$$

If LTE is verified, the plot is a line with $1 / k T$ slope. Boltzmann plots for a cold plasma produced in PSEG are displayed in Fig. 9. As there exist no emission lines corresponding to energy levels above ${ }^{2} \mathrm{P}_{1}$, we carried out a least-square fitting to scarce data points, which results into a low coefficient of determination less than 0.2. For each pressure case, no ionized states of argon were inferred 


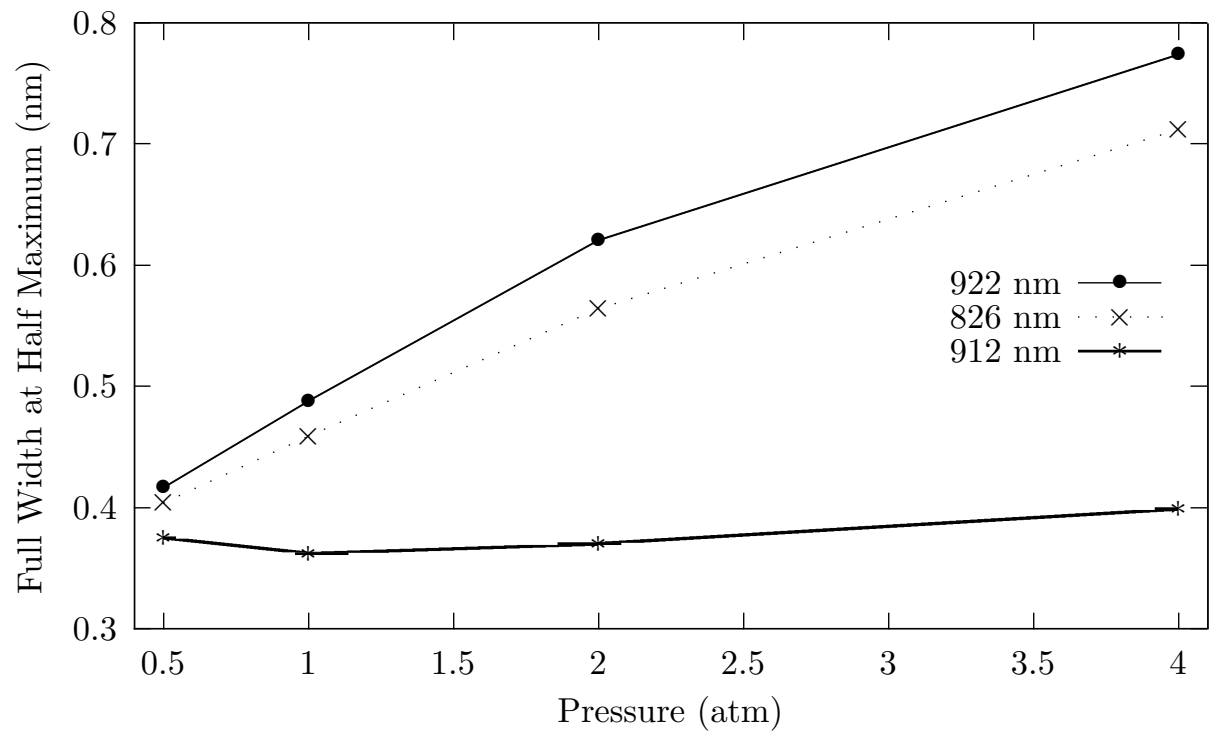

Figure 8: FWHM of argon emission lines at $826 \mathrm{~nm}, 922 \mathrm{~nm}$ (resonant ${ }^{1} \mathrm{~S}_{2}$-linked levels) and $912 \mathrm{~nm}$ (optical level). The instrument spectral resolution was $0.36 \mathrm{~nm}$.

with confidence since one can not estimate any positive slope. This way, no LTE conditions holds. In other words, other models are needed for understanding the plasma light emission.

\section{Conclusion}

The present paper has detailed the definition, manufacture and validation of an innovative testing device, named PSEG (Prototype of Scintillator ElectrodesGas), dedicated to a long-term endeavor to develop dependable optical fission chambers for pool-type Generation IV SFR. Main PSEG mission is to produce spectral emission data in steady state and pollution-free conditions by $\mathrm{MeV}$-range heavy ions to be compared with cold-plasma simulations and test technological components for light detection.

An alpha-particle source was used to mock-up a noble-gas plasma as it will 

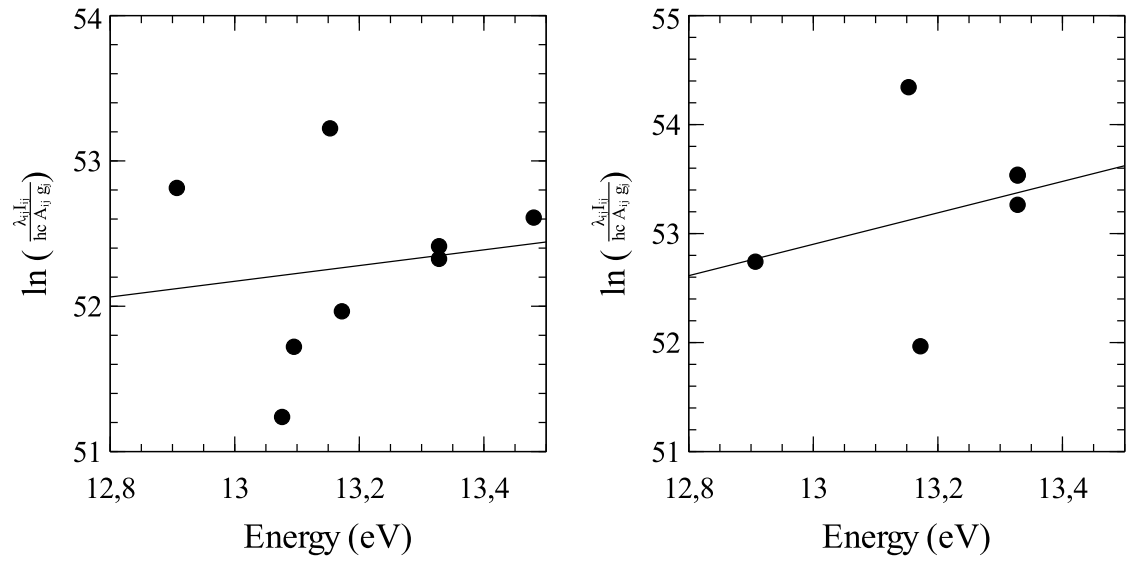

Figure 9: Boltzmann plots for a cold-plasma excited by alpha particles at 0.5 atm (left) and $4 \mathrm{~atm}$ (right).

be produced in future optical fission chambers. OES of argon and helium at various pressures and room temperature indicated strong atomic emission lines. The absence of continuum on all the experimental spectra unveils low ionization rates and electronic densities. Boltzmann plots showed that no LTE was established. Moreover, a linear relationship between the FWMH of some resonancebroadened emission lines and the PSEG pressure was proven. This comes to promising self-diagnosis capabilities of optical fission chambers.

\section{References}

[1] C. Jammes, N. Chapoutier, P. Filliatre, J.-P. Jeannot, F. Jadot, D. Verrier, A.-C. Scholer, B. Bernardin, Neutron flux monitoring system of the french gen-iv sfr: Assessment of diverse solutions for in-vessel detector installation, Nuclear Engineering and Design 270 (2014) 273 - 282. doi:https://doi.org/10.1016/j.nucengdes.2013.12.057.

URL http://www.sciencedirect.com/science/article/pii/ S0029549314000338

[2] C. Jammes, P. Filliatre, B. Geslot, T. Domenech, S. Normand, Assessment 
of the high temperature fission chamber technology for the french fast reactor program, IEEE Transactions on Nuclear Science 59 (2011) 1351-1359.

[3] G. Galli, Study of partial partial-discharge-induced pulses in a high temperature fission chambers, Ph.D thesis, Université Paris-Saclay (Dec. 2018).

URL https://tel.archives-ouvertes.fr/tel-02170517

[4] G. Cheymol, H. Long, J. F. Villard, B. Brichard, High level gamma and neutron irradiation of silica optical fibers in cea osiris nuclear reactor, IEEE Transactions on Nuclear Science 55 (4) (2008) 2252-2258. doi:10.1109/TNS.2008.924056.

[5] G. Laffont, R. Cotillard, N. Roussel, R. Desmarchelier, S. Rougeault, Temperature resistant fiber bragg gratings for on-line and structural health monitoring of the next-generation of nuclear reactors, Sensors 18 (2018) 1791. doi:10.3390/s18061791.

[6] P. Ferdinand, S. Magne, G. Laffont, Optical fiber sensors to improve the safety of nuclear power plants, Fourth Asia Pacific Optical Sensors Conference, International Society for Optics and Photonics, Vol. 8924, 2013. doi:10.1117/12.2033922.

URL https://doi.org/10.1117/12.2033922

[7] A. I. Nadeev, I. A. Razenkov, D. I. Shelefontyuk, E. S. Shevtsov, Comparison of an avalanche photodiode and a photomultiplier tube as photodetectors of near-infrared radiation in the photon-counting mode, Instruments and Experimental Techniques 61 (3) (2018) 377-381. doi:10.1134/S0020441218020057.

URL https://doi.org/10.1134/S0020441218020057

[8] C. Eggler, C. Huddleston, Gaseous Scintilliation. IRE Transactions on Nuclear Sciences 3(4) 1956 36-38.

[9] E. Grün, A: Schoepper, Über die fluoreszenz von gasen bei anregung durch a-teilchen, Naturforsch 6a (1951) 698. 
[10] L. Koch, Étude de la fluorescence des gaz rares excités par des particules nucléaires. Utilisation pour la détection des rayonnements nucléaires., Ph.D. thesis, Université de Paris (1959).

[11] G. Miley, P. Thiess, A unified approach to two region ionization-excitation density calculations, Nuclear Applications 6 (5) (1969) 435-451.

[12] J. C. Guyot, G. H. Miley, J. T. Verdeyen, Application of a tworegion heavy charged particle model to noble-gas plasmas induced by nuclear radiations, Nuclear Science and Engineering 48 (4) (1972) 373386. arXiv:https://doi.org/10.13182/NSE72-A22505, doi:10.13182/NSE72A22505.

URL https://doi.org/10.13182/NSE72-A22505

[13] U. Giesen, J. Beck, New measurements of W-values for protons and alpha particles, Radiation Protection Dosimetry 161 (1-4) (2014) 23-26. doi:10.1093/rpd/nct282.

[14] M. K. Chung, A \& Prelas, Charged particle spectra from U235 and B10 micropellets and slab coatings, Laser and Particle Beams 2 (2) (1984) 201211.

[15] J. F. Ziegler, M. D. Ziegler, J. P. Biersack, SRIM - The stopping and range of ions in matter (2010), Nuclear Instruments and Methods in Physics Research B 268 (2010) 1818-1823. doi:10.1016/j.nimb.2010.02.091.

[16] V. Nitzki, H. Matzke, Stopping power of 1-9-Mev $\mathrm{He}++$ ions in UO2,(U, $\mathrm{Pu}) \mathrm{O} 2$, and ThO2, Physical Review B 8 (5) (1973) 1894.

[17] M. Saeki, T. Ishimori, Ranges of fission fragments in uranium-aluminum alloys and uranium oxides., Nippon Genshiryoku Gakkaishi 14(6) (1972) $278-282$.

[18] R. Platzman, Total ionization in gases by high-energy particles: An appraisal of our understanding, The International Journal of Applied Radiation and Isotopes 10 (2) (1961) 116 - 127. 
doi:https://doi.org/10.1016/0020-708X(61)90108-9.

URL http://www.sciencedirect.com/science/article/pii/ $0020708 \times 61901089$

[19] R. Chandrasekharan, M. Messina, A. Rubbia, Detection of noble gas scintillation light with large area avalanche photodiodes (LAAPDS), Nuclear Instruments and Methods in Physics Research Section A: Accelerators, Spectrometers, Detectors and Associated Equipment 546 (3) (2005) 426 437. doi:https://doi.org/10.1016/j.nima.2005.03.105.

URL http://www.sciencedirect.com/science/article/pii/ S0168900205007655

[20] M. J. Kushner, Response times and energy partitioning in electronbeam-excited plasmas, Journal of Applied Physics 66 (1989) 2297-2306. doi:10.1063/1.344286.

[21] J. Birks, The Theory and Practice of Scintillation Counting, Elsevier, 2013.

[22] LL. Alves, The IST-LISBON database on LXCat, Journal of Physics Conference Series 565 (1).

[23] G.W.F Drake,Molecular and Optical physics handbook , American Institute of Physics, Woodbury, NY 1996. 